\title{
VEINTICINCO AÑOS DE MEJORAMIENTO EN LOS SISTEMAS DE MAÍZ EN CENTROAMÉRICA: LOGROS Y ESTRATEGIAS HACIA EL AÑO 20001
}

\author{
Hugo Córdova ${ }^{2}$, Salvador Castellanos ${ }^{2}$, Héctor Barreto ${ }^{2}$, Jorge Bolaños $^{3}$
}

\begin{abstract}
RESUMEN
Veinticinco años de mejoramiento en los sistemas de maíz en Centroamérica: logros y estrategias hacia el año 2000. La ciencia y la tecnología constituyen la brecha más importante entre la pobreza y la prosperidad. Durante el último cuarto de siglo, la introducción de prácticas tecnológicas basadas en la ciencia, han ayudado a muchos países en desarrollo a alcanzar una relación favorable entre la producción de alimentos y el crecimiento de la población. En Centroamérica y El Caribe el mejoramiento genético del maíz ha contribuido a la liberación de aproximadamente 80 cultivares que han sido multiplicados y distribuidos por una industria de semillas dinámica y progresista. Estos cultivares, asociados con prácticas agronómicas apropiadas, han permitido aumentar la productividad del maíz en la región, lo que ha representado el incremento del rendimiento en un $60 \%$ aproximadamente, siendo este aspecto más significativo en países como El Salvador y Guatemala que duplicaron los rendimientos y mejoraron el autoabastecimiento de maíz. Los cultivares tolerantes al achaparramiento y adaptados a condiciones de humedad limitada, están desempeñando un papel muy importante en el mantenimiento sustentable de la producción. La liberación de híbridos modernos de maíz, tolerantes a factores adversos, bióticos y abióticos, contribuirá a reducir las pérdidas postcosecha. El mejor entendimiento de algunos aspectos fisiológicos correlacionados con el rendimiento y la incorporación de índices de selección más eficientes en las metodologías de mejoramiento para ambientes adversos, han mejorado las técnicas de selección.
\end{abstract}

\begin{abstract}
Twenty five years of system's improvement of corn in Central America: accomplishments and strategies toward the year 2000. Science and technology constitute the most important bridge between prosperity and poverty. During the last quarter of the century, the introduction of modern technology based on science, has assisted developed countries to reach a favorable balance between food production and population growth. In Central America, corn breeding has contributed to the release of near a hundred varieties that have been multiplied and distributed by a dynamic and progressive seed industry. These new maize hybrids associated with the proper agronomy practices have increased maize productivity in the region by $60 \%$ yield in the past 15 years. This is certainly a fact in El Salvador and Guatemala that duplicated the yields and self-sufficiency in corn. New varieties resistant to biotic and abiotic stresses are playing a very important role in the sustainability of maize production. The future release of new modern hybrids resistant to such stresses will contribute to reduce the post-harvest losses. The better understanding of some physiological aspects correlated with yield and more effective methods of selection in maize breeding have contributed to improve the selection techniques, however, the weakness of the national research and extension programs, the lack of economic resources to agricultural production and seed industry and the deterioration of the natural resources call for a new integrated regional effort. This new effort should be focussed in the sustainable production that guarantees the increase in productivity in the next two decades.
\end{abstract}

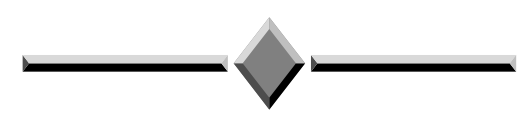

\section{INTRODUCCIÓN}

Existe evidencia abrumadora de que el cambio de siglo exige la definición e implementación de políticas de desarrollo congruentes con el crecimiento de la po- blación, la conservación de los recursos naturales y la seguridad alimentaria. Dentro de este escenario, la política de desarrollo de investigación agrícola urgirá el diseño de estrategias de investigación, orientadas al uso eficiente de los recursos genéticos para alimentar a una

\footnotetext{
1 Recibido para publicación el 5 de octubre del 2001.

2 Centro Internacional de Mejoramiento de Maíz y Trigo (CIMMYT). Apdo. Postal 6-641 CP 06600 México, D.F. México. E-mail: cimmyt@ggnet.com.

3 Programa Regional de Maíz, CIMMYT Guatemala.
} 
población de centroamericanos que crece a una tasa anual de 2,5\%; por lo tanto, habrá 70 millones de centroamericanos cuando ocurra el cambio de siglo.

El esfuerzo conjunto por parte de los programas nacionales y la empresa privada regional para el desarrollo y transferencia de nuevas alternativas de producción han contribuido a mejorar el autoabastecimiento de maíz en la región centroamericana. Sin embargo, el debilitamiento de los programas nacionales de investigación, del soporte de transferencia de tecnología agrícola, del apoyo financiero a la producción agrícola, de la industria de semillas, así como el deterioro de los recursos naturales, demandan un nuevo esfuerzo integrado en la colaboración regional. Éste debe desplegarse con un enfoque de producción sostenible que garantice el incremento de la productividad en las próximas décadas, a través del manejo eficiente de cultivos y con énfasis en el desarrollo de cultivares resistentes a factores adversos bióticos y abióticos adaptados a ambientes marginales, en donde existe la expectativa de que la biotecnología aplicada apropiadamente, contribuya al mejoramiento genético convencional en los próximos diez años.

En este documento se discuten los métodos de mejoramiento genético utilizados, se destacan los logros regionales y se sugieren estrategias pertinentes al mantenimiento de la productividad del cultivo de maíz en la próxima década.

\section{EVOLUCIÓN DEL ENFOQUE REGIONAL DE MEJORAMIENTO GENÉTICO DE MAÍZ}

\section{Evolución de los métodos de mejoramiento genético}

En la década de los 70's se puso de moda el uso de métodos de selección recurrente intra-poblacional, los cuales ponen énfasis en la capitalización de efectos genéticos aditivos (heredables), a través de una secuencia cíclica en la cual se obtenía en cada ciclo el mejoramiento de la población y a la vez se extraía una nueva variedad mejorada de la fracción superior de familias seleccionadas; la presión de selección ejercida fue en promedio el $20 \%$ para continuar el ciclo de mejoramiento y $2,5 \%$ para formar el compuesto que constituyó la variedad experimental. Las fuentes de germoplasma usadas fueron obtenidas del CIMMYT.

Los métodos usados en los primeros años de la década de los 70's incluyeron básicamente selección masal y de mazorca por surco, utilizándose muy poco métodos que incluían endogamia (autofecundación) en el proceso. El único país que usó efectivamente la selección genealógica para el desarrollo de líneas endogámicas e híbridos fue El Salvador, especialmente utilizando poblaciones $\mathrm{F}_{2}$ de cruzas simples o dobles para el reciclaje de líneas, permitiendo la liberación de los híbridos H-3 en 1960 y H-5 en 1965.

La mayoría de las variedades de maíz de polinización libre liberadas en el quinquenio 1970 - 1975, fueron producto de la selección masal o mazorca por surco.

En la segunda mitad de los años 70's se incorporan cambios muy importantes en el mejoramiento de maíz en Centroamérica tales como la incorporación que hace el CIMMYT en sus poblaciones del método de selección recurrente de progenitores de hermanos completos (HC), usando progenies entre cruzas directas y recíprocas para su evaluación en ensayos internacionales de progenies (IPTT); básicamente los programas nacionales adoptan esta metodología o variaciones de la misma, con la cual se esperaba capitalizar $1 / 2$ de la varianza aditiva y $1 / 4$ de la varianza de dominancia presente entre progenies. Las variedades liberadas durante éste segundo quinquenio de la década de los 70's, fueron desarrolladas usando esta metodología. El CIMMYT inicia el Programa Regional para Centroamérica y El Caribe básicamente con el mismo enfoque:

1. Método de selección recurrente de progenies de hermanos carnales.

2. Los consultores del CIMMYT de este programa sugieren una metodología combinada de líneas $\mathrm{S}_{1}$ y progenies de medios hermanos $(\mathrm{MH})$ en algunos países (Costa Rica).

Los métodos de mejoramiento genético del cultivo de maíz en Centro América, evolucionaron paralelamente con la capacitación obtenida por los fitomejoradores de los programas nacionales responsables del desarrollo de cultivares y al asesoramiento recibido del exterior (principalmente de CIMMYT). Esta influencia externa limitó, en parte, la pronta evolución de los métodos de selección intra-familiar, usando familias de vigor completo a métodos que involucren endogamia drástica (autofecundación) en el proceso de selección y como consecuencia a métodos de hibridación convencional.

El Cuadro 1 presenta los cambios ocurridos en las metodologías de fitomejoramiento de maíz en Centroamérica de 1970 a 1995 . Nótese que los países que más pronto adoptaron el desarrollo de líneas endogámicas (hibridación) en sus métodos de mejoramiento, han sido los más exitosos en el desarrollo de cultivares. 
Cuadro 1. Evolución de los métodos de mejoramiento en Centroamérica.

\begin{tabular}{llll}
\hline \multicolumn{1}{c}{ País } & \multicolumn{1}{c}{$\mathbf{1 9 7 0 - 1 9 8 0}$} & $\mathbf{1 9 8 1 - 1 9 9 0}$ & \multicolumn{1}{c}{$\mathbf{1 9 9 1 - 1 9 9 5}$} \\
\hline El Salvador & Hibridación & Hibridación & Hibridación \\
Guatemala & SM-SMH-SHC-HI & Hibridación & Hibridación \\
Honduras & SM-SMH-SHC & SHC-S1 & LS1 Hibridación \\
Nicaragua & SM-SMH-SHC & SMH-S1-HI & LS1 Hibridación \\
Costa Rica & MH-S1MH & S1MH-HI & -- \\
Panamá & SM-MH & SM-MH-HI & Hibridación \\
\hline
\end{tabular}

$\mathrm{SM}=$ selección masal, $\mathrm{MH}=$ medios hermanos, $\mathrm{HC}=$ hermanos carnales.

$\mathrm{LS}_{1}=$ líneas $\mathrm{S}_{1}$, HI = hibridación

\section{Fuentes de germoplasma}

La elección correcta de la fuente de germoplasma significa el $50 \%$ del éxito en un programa moderno de mejoramiento genético, dependiendo el otro $50 \%$ en el desarrollo eficiente de líneas para la formación de híbridos y sintéticos.

Las fuentes de germoplasma usadas por los programas nacionales de maíz de Centroamérica, fueron en más del 90\% de los casos, provenientes de CIMMYT (antes Oficina de Estudios Especiales, que posteriormente dio origen a CIMMYT). A finales de los años 50's y 60's, la Oficina de Estudios Especiales en México en colaboración con INIA (Gobierno de México), desarrollaron líneas de diferentes fuentes tropicales, las cuales estuvieron disponibles para todos los fitomejoradores de los programas nacionales; dependiendo su uso eficiente, de la iniciativa de estos profesionales, siendo un buen ejemplo las tres líneas que formaron parte del híbrido H-5. Otra fuente de germoplasma (aunque muy poco utilizada) fue la generación de poblaciones $\mathrm{F}_{2}$ de híbridos ya liberados por la industria semillista privada para el reciclaje de líneas a través de autofecundaciones sucesivas por selección genealógica, siendo un ejemplo las tres líneas del híbrido salvadoreño H-3, las cuales fueron derivadas de los híbridos T-23 y T-25 de origen Semillas Poey. Otra vez se observa que la iniciativa del mejorador hace uso eficiente del germoplama apropiado disponible.

El reciclaje eficiente de líneas de híbridos de alto potencial sigue siendo en la actualidad el método más usado por la industria privada. Las fuentes de germoplasma originadas en CIMMYT más usadas fueron: Tuxpeño-1, La Posta, Mezcla Tropical Blanca, Tuxpeño Caribe y Eto blanco.

\section{Caracteres seleccionados}

Los criterios de selección más importantes usados para mejorar la estabilidad del rendimiento han sido: alto potencial de rendimiento, resistencia a pudrición de mazorca, resistencia al acame, altura de planta y mazorca adecuada, resistencia a sequía y achaparramiento.

La implementación del programa de híbridos en CIMMYT durante 1986, ha permitido el desarrollo de líneas endogámicas sobresalientes y el desarrollo de híbridos adaptados a ambientes tropicales. Estos progenitores en combinación con las líneas de los algunos programas nacionales de El Salvador, Honduras y Guatemala, han dado origen a nuevas combinaciones híbridas que han sido liberadas por los programas nacionales mencionados. En Panamá y Costa Rica se liberaron híbridos cuyos progenitores son totalmente originales del programa de híbridos tropicales de CIMMYT.

Definitivamente, el impacto de la endogámica se ha hecho presente en los programas nacionales de mejoramiento genético de Centroamérica y El Caribe, $100 \%$ de ellos están usando métodos de mejoramiento que involucran endogamia en el proceso y solamente el $18 \%$ (dos programas) usaron $\mathrm{MH}$ como método de mejoramiento durante 1996.

\section{Evolución del enfoque regional}

El Programa Regional de Maíz para Centroamérica y El Caribe, red regional de investigación en maíz formalmente establecido en 1974 y que es financiado por la Cooperación Suiza para el Desarrollo (COSU$\mathrm{DE})$, tiene como objetivo principal contribuir a incrementar la producción y la productividad del cultivo del maíz en la región. El proyecto ha fomentado este objetivo a través del fortalecimiento de las instituciones nacionales de investigación, promoviendo en cada país el desarrollo de sistemas integrados de mejoramiento y producción apoyados por entrenamiento dinámico y consultas en fitomejoramiento, agronomía y producción de semillas. El esfuerzo realizado por los programas nacionales en el desarrollo de nuevas alternativas de producción ha contribuido a mejorar la autosuficiencia en abastecimiento de maíz en los países de Centroamérica y El Caribe. Este enfoque duró de 1975 - 1985 y estuvo fundamentado en consultorías, distribución de germoplasma y en entrenamiento de personal.

En 1986 se inicia un período en que la colaboración horizontal establece sus raíces profundas y el agricultor de zonas marginales es el elemento central del proceso de generación prueba y transferencia de tecno- 
logía. Esta red de colaboración horizontal se le conoce ahora como Programa Regional de Maíz (PRM).

\section{UNA DÉCADA DE COLABORACIÓN HORIZON- TAL HACIA LA PRODUCCIÓN SOSTENIBLE}

\section{Establecimiento de prioridades}

La identificación de los factores limitantes de la producción de maíz y el establecimiento de prioridades de investigación ha permitido diseñar proyectos colaborativos regionales en agronomía, desarrollo y mejoramiento de germoplasma orientados a resolver dichos factores adversos. El establecimiento de prioridades se realiza con base en un proceso interactivo en el cual se discriminan los factores limitantes de la producción ordenados en términos de prioridades.

Desarrollo y mejoramiento de germoplasma de maíz resistente al achaparramiento

Uno de los resultados más sobresalientes de la colaboración horizontal entre los programas nacionales de Centroamérica, El Caribe y CIMMYT es el Proyecto Colaborativo de Desarrollo de Cultivares resistentes al Achaparramiento. Los responsables de este proyecto son El Salvador, Nicaragua y República Dominicana.

Los cultivares desarrollados en este proyecto están contribuyendo a incrementar la productividad del cultivo del maíz en varios países de Latinoamérica. En Nicaragua en particular, hay evidencias muy notables de la respuesta de estos cultivares ante la incidencia del micoplasma que es el organismo causante de esta enfer- medad. Estudios nacionales indican que la variedad tolerante NB-6 sembrada en 2000 hectáreas rindió 3,5 t/ha, en tanto que los híbridos susceptibles al achaparramiento sólo rindieron 1,5 t/ha.

El Cuadro 2 muestra el progreso logrado. Nótese que la variedad sintética derivada del ciclo tres de mejoramiento de la población 73 es superior en rendimiento y resistencia al achaparramiento que la variedad Santa Rosa 8073 (ciclo 0). Esta superioridad indica un progreso del $24,88 \%$, para rendimiento de grano que equivale a un incremento de $846 \mathrm{~kg} / \mathrm{ha}$, lo que puede interpretarse como un $8,29 \%$ ó $282 \mathrm{~kg} / \mathrm{ha}$, por ciclo de mejoramiento. Este incremento en rendimiento está asociado a un incremento considerable en resistencia al achaparramiento, que resulta en una reducción en la enfermedad de 12,5\%. Estos resultados son más relevantes cuando hacemos la comparación con el testigo susceptible H-5. El sintético SC3 P73 rindió 4246 kg/ha con $16,6 \%$ de mazorcas afectadas por la enfermedad del achaparramiento y el testigo susceptible rindió 2549 $\mathrm{kg} / \mathrm{ha}$ con $53 \%$ de mazorcas afectadas. Los resultados mostrados incluyen ambientes cuya presencia de la enfermedad varió de severa a óptima, indicando que los agricultores reducirían o disminuirían sus pérdidas al adoptar los nuevos sintéticos desarrollados. Estos logros superan las expectativas que se establecieron en los indicadores verificables (Córdova 1990, Aguiluz et al. 1990).

Desarrollo y mejoramiento de germoplasma adaptado a condiciones de humedad limitada

La tendencia de cierta fracción de agricultores en los países de Centroamérica es de sembrar maíz en ambientes marginales de producción. Gradualmente el

Cuadro 2. Promedios de rendimiento y características agronómicas de 10 genotipos de maíz resistentes al achaparramiento evaluados en siete ambientes de Centroamérica y El Caribe. 1990.

\begin{tabular}{lccccccc}
\hline \multicolumn{1}{c}{ Variedad } & $\begin{array}{c}\text { Rend. } \\
\text { kg/ha }\end{array}$ & $\begin{array}{c}\text { \% } \\
\text { H-5 }\end{array}$ & $\begin{array}{c}\text { Días } \\
\text { Flor. }\end{array}$ & $\begin{array}{c}\text { Alt. } \\
\text { Mzca. }\end{array}$ & $\begin{array}{c}\text { Asp. } \\
\text { Mzca. }\end{array}$ & $\begin{array}{c}\text { \% Mzca. \% Plantas } \\
\text { Afect. }\end{array}$ & $\begin{array}{c}\text { Achap. } \\
\text { SC3P73 }\end{array}$ \\
\hline (8x4) (7x2) & 4246 & 166 & 54 & 122 & 2,5 & 16,6 & 25,1 \\
H-53 & 4121 & 162 & 56 & 130 & 2,5 & 26,6 & 32,7 \\
NB-12 & 4056 & 159 & 54 & 120 & 2,5 & 28,3 & 33,2 \\
(9x15) (8x7) & 3941 & 154 & 55 & 126 & 2,5 & 16,5 & 32,6 \\
Sint, STR36C3 & 3841 & 150 & 55 & 121 & 3,0 & 34,3 & 34,5 \\
NB-6 & 3597 & 141 & 54 & 108 & 3,0 & 32,2 & 37,7 \\
Santa Rosa 8073 & 3510 & 137 & 54 & 119 & 3,0 & 29,7 & 38,1 \\
CESDA 88 & 3112 & 133 & 55 & 115 & 3,0 & 29,1 & 37,5 \\
H-5 & 2549 & 100 & 57 & 115 & 3,0 & 24,0 & 45,8 \\
\hline
\end{tabular}


maíz está siendo sustituido de las áreas con potencial económico por otros cultivos con mayor rentabilidad.

Uno de los factores adversos abióticos más importante de estas áreas marginales es la sequía, que afecta anualmente las áreas productoras de maíz que generalmente son caracterizadas por condiciones de pendiente, suelos superficiales y con poca capacidad de retención de humedad.

La respuesta diferencial del germoplasma de maíz desarrollado bajo presión de factores adversos, puede contribuir a la estabilidad de los genotipos a la mayoría de ambientes de recomendación.

Esta condición de ambiente de humedad limitada es aplicada artificialmente para selección por parte del Subproyecto Colaborativo de Sequía del PRM. También se involucra dentro de la metodología de mejoramiento, una evaluación de las progenies bajo condiciones de precipitación pluvial errática para lograr la selección de los alelos favorables que condicionan la adaptación de este germoplasma a los ambientes diversos de producción.

La Figura 1 muestra el comportamiento de la variedad BS-16 tolerante a condiciones de humedad limitada, evaluada bajo ambientes de sequía durante la floración. Es notable su comportamiento bajo estas circunstancias ya que su rendimiento $3900 \mathrm{~kg} / \mathrm{ha}$, duplica el rendimiento de la variedad local de $1800 \mathrm{~kg} / \mathrm{ha}$; así mismo, el índice de resistencia a sequía es 0,71 comparado con 0,54 de la variedad local, lo que indica la eficiencia del mejoramiento para estas condiciones (Brizuela et al. 1990, Córdova 1990).

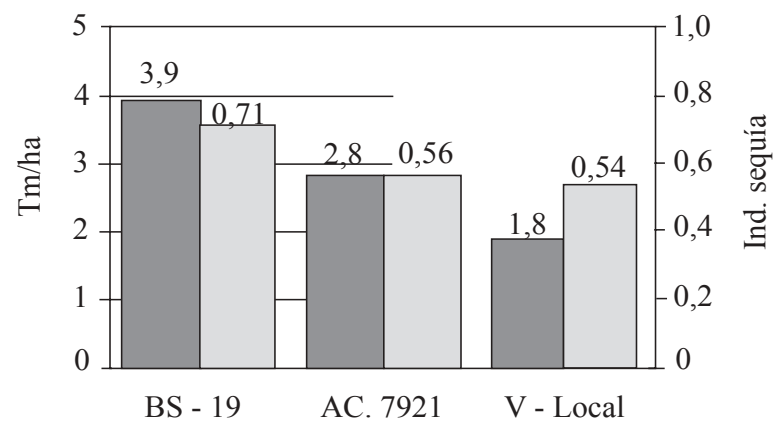

Rendimiento $\square$ Ind. sequía

Figura 1. Efecto de la selección para tolerancia a sequía.
Desarrollo y mejoramiento de germoplasma de maíz resistente a pudrición de mazorca

Los agricultores que siembran maíz bajo condiciones de alta precipitación pluvial, en la Costa Atlántica de Honduras, Costa Rica y Guatemala, dejan el maíz en el campo por largos períodos después de haber alcanzado la madurez fisiológica. Esta circunstancia ocasiona el deterioro del cultivo causando pudrición de la mazorca y el tallo, producida por los hongos Diplodia sp. y Fusarium spp. Para dar respuesta a este factor adverso se estableció el subproyecto colaborativo de mejoramiento de la resistencia a pudrición de mazorca cuyo líder es Costa Rica. En 1987 se establece la metodología de selección recurrente de líneas $\mathrm{S}_{1}$ como estrategia de mejoramiento poblacional. Para la evaluación de progenies de líneas $\mathrm{S}_{1}$ se utilizan dos localidades de Costa Rica con presión de inóculo natural y una localidad de Honduras en donde se realizan inoculaciones artificiales con Diplodia.

Las progenies seleccionadas para formar la variedad sintética Diamante 9043 tolerante a pudrición de mazorca mostraron una media de pudrición de mazorca del $11 \%$ y un incremento en rendimiento del $17 \%$ en tanto que la media de la población (testigo) mostró $32,9 \%$ de mazorcas podridas, reduciéndose en un $21 \%$ el efecto de ese factor adverso biótico. Resultados similares se obtuvieron en la variedad sintética RPM x Tuxp C17-90 con un incremento en rendimiento del $32 \%$.

Los diferenciales de selección permiten:

a) Reducir los problemas de mala cobertura en un ciclo avanzado de selección de la población RPM x Tux PC17-90 y en un grupo de líneas derivadas de la variedad Diamantes 8043 (población La Posta).

b) Eliminar la frecuencia de genes recesivos deletereos presentes en las poblaciones antes mencionadas para aumentar la ganancia en rendimiento.

c) Incrementar la frecuencia de alelos favorables involucrados en resistencia a pudrición de tallo y mazorca.

d) Desarrollar variedades sintéticas y/o híbridos de buena cobertura y resistencia a pudrición de tallo y mazorca (Jiménez et al. 1990, Córdova 1990).

Desarrollo y mejoramiento de híbridos con adaptación a Centroamérica y El Caribe

El progreso en el mejoramiento del cultivo del maíz por hibridación en Centroamérica ha sido muy evidente en los últimos 10 años, habiéndose logrado 
una ganancia por año de 3,5\% de incremento en rendimiento, así como en tolerancia a factores adversos bióticos y abióticos (Córdova 1991).

Cuadro 3. Cultivares resistentes a condiciones de humedad limitada liberados en Centro América, 1987 - 1991.

\begin{tabular}{lll}
\multicolumn{1}{c}{ Cultivar } & \multicolumn{1}{c}{ País } & \multicolumn{1}{c}{ Características } \\
\hline BS-19 & Guatemala & B.D.T.T. \\
B-5 & Guatemala & B.D.T.I. \\
Chorotega & Honduras & B.D.T.I. \\
Pasaquina & El Salvador & B.D.T.T. \\
\hline
\end{tabular}

Cultivares resistentes al achaparramiento liberados en Mesoamérica y Venezuela, 1987-1991.

\begin{tabular}{lll}
\hline \multicolumn{1}{c}{ Cultivar } & \multicolumn{1}{c}{ País } & \multicolumn{1}{c}{ Características } \\
NB-6M & Nicaragua & B. Tolerante al achaparramiento \\
NB-12 & Nicaragua & B. Tolerante al achaparramiento \\
La Lujosa & Honduras & B. D. Tolerante \\
CESDA 88 & República & A. Tolerante \\
& Dominicana & \\
H-53 & El Salvador & Resistente \\
S.R. 8073 & Venezuela & B. Tolerante \\
S.R. 8073 & México & B. Tolerante \\
\hline
\end{tabular}

Híbridos de maíz liberados en Centro América y El Caribe, 1980.

\begin{tabular}{|c|c|c|}
\hline Híbridos & País & Características \\
\hline $\mathrm{H}-30$ & Honduras & Tolerante a pudrición de mazorca \\
\hline P-8812 & Panamá & Tolerante a pudrición de mazorca \\
\hline P-8814 & Panamá & Tolerante a pudrición de mazorca \\
\hline P-8916 & Panamá & Tolerante a pudrición de mazorca \\
\hline HC-43 & Costa Rica & Tolerante a pudrición de mazorca \\
\hline $\mathrm{HN}-876$ & Nicaragua & Tolerante a pudrición de mazorca \\
\hline H-104, H-9 & El Salvador & Tolerante a pudrición de mazorca \\
\hline $\mathrm{H}-57$ & El Salvador & Tolerante a pudrición de mazorca \\
\hline $\begin{array}{l}\text { HB-11, HS-83, } \\
\text { H-685 }\end{array}$ & Guatemala & Tolerante a pudrición de mazorca \\
\hline
\end{tabular}

En 1990 se condujeron 13 ensayos en Centroamérica y República Dominicana, con el objetivo de evaluar los híbridos superiores identificados en 1989, con mayor estabilidad y respuesta diferencial a ambientes contrastantes.

El híbrido Exp. 901 rindió $6000 \mathrm{~kg} / \mathrm{ha}$ a través de trece localidades de Centroamérica, superando al testigo H-5 en un $23 \%$. En la localidad de Cuyuta, donde existió la presencia más alta del estrés biótico de pudrición de mazorca, este híbrido rindió $4000 \mathrm{~kg} / \mathrm{ha}$ con sólo el $9 \%$ de mazorcas podridas y un rendimiento de grano limpio de $3640 \mathrm{~kg} / \mathrm{ha}$. Bajo condiciones óptimas Exp. 901 rindió 9,2 t/ha en tanto que H-5 rindió 25\% menos (Figura 2).

\section{IMPACTO DEL MEJORAMIENTO GENÉTICO}

Es difícil dimensionar y establecer la magnitud del impacto que un programa regional puede causar. Sin embargo, se pueden establecer algunas medidas que bien implementadas podrían dar a conocer los beneficios y logros de un trabajo muy dinámico, con objetivos precisos y bien definidos.

\section{Liberación de cultivares}

La orientación en el mejoramiento para resistencia a factores adversos bióticos y abióticos para adaptación a ambientes marginales, como una respuesta al pequeño agricultor ha permitido liberar más de 100 híbridos y variedades de maíz en los últimos veinticinco años. Tanto híbridos como variedades liberadas en 1989, 1990 y 1991 presentan características descriptivas que los hacen competir con ventaja en los ambientes desfavorables. Estas ventajas comparativas reducen el riesgo que el agricultor atraviesa al realizar su pequeña inversión, principalmente en fertilizantes.

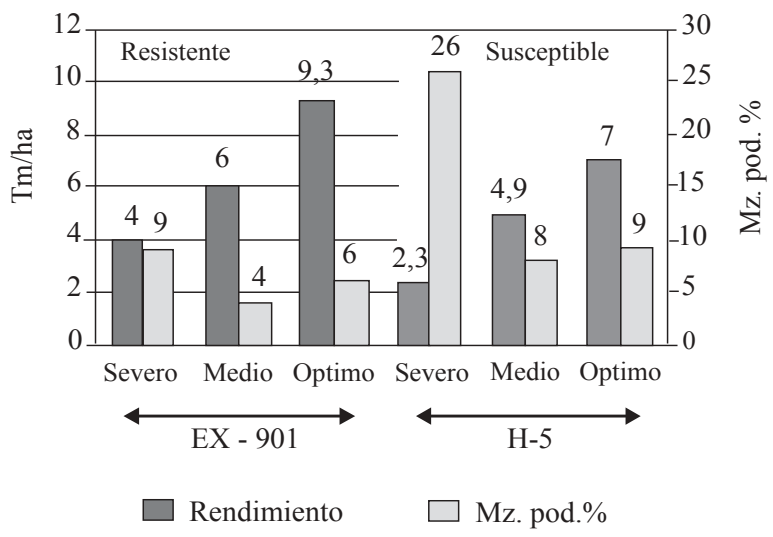

Figura 2. Efecto del mejoramiento para resistencia a pudrición de mazorca sobre el rendimiento de maíz.

\section{Progreso en el mejoramiento genético}

Las Figuras 4, 5 y 6 muestran el avance alcanzado por los Programas Nacionales como consecuencia de la capacitación en fitomejoramiento, siendo este progreso más evidente en Guatemala, El Salvador, Honduras y Nicaragua, lográndose un avance notable del $27 \%$ en mejoramiento, que equivale al $30 \%$ de incremento en rendimiento y resistencia a factores adversos bióticos y abióticos (Cuadro 4). 
Cuadro 4. Medias de rendimiento y parámetros de estabilidad del rendimiento de híbridos de maíz evaluados en 52 ambientes de Norte América, Centroamérica, El Caribe y Venezuela. 1988-1990.

\begin{tabular}{lcccl}
\hline Híbridos & Tm/ha & \% de H-5 & $\beta_{\mathbf{l}}$ & $\mathbf{S}_{\mathbf{d l}}{ }^{2}$ \\
\hline HB-85 & 6,00 & 127 & 1,10 & 0,076 \\
HB-83 & 5,73 & 122 & 1,06 & 0,067 \\
H-30 & 5,72 & 122 & $1,22^{* *}$ & 0,088 \\
MAX 307 & 5,55 & 117 & 0,93 & $0,155^{*}$ \\
HA-46 & 5,53 & 117 & 1,07 & $0,160^{*}$ \\
XCH-53 & 5,34 & 113 & 1,01 & $0,230^{* *}$ \\
HR-17 & 5,22 & 110 & 1,12 & 0,050 \\
H-5 & 4,72 & 100 & 1,09 & 0,085 \\
MDSH & 0,403 & & & \\
\hline
\end{tabular}

\section{Incremento en los rendimientos}

El desarrollo de alternativas tecnológicas de producción permitió que el rendimiento se incrementara en un 55\% entre 1975 y 1988. Los países que más contribuyeron a este incremento son: Guatemala, El Salvador y Nicaragua (Cuadro 5). Sin embargo, estos incrementos son muy frágiles si no se toman medidas que estimulen la producción en las áreas rurales. La Figura 7 muestra las tendencias de la demanda de producción y rendimientos de maíz en Guatemala hasta el año 2000. Las estimaciones fueron realizadas haciendo un análisis de regresión basado en tendencias de la producción hasta 1988.

Cuadro 5. Superficie y rendimiento de maíz en Centroamérica y El Caribe. 1975-1988.

\begin{tabular}{|c|c|c|c|c|}
\hline \multirow[b]{2}{*}{ País } & \multicolumn{2}{|c|}{ 1975* } & \multicolumn{2}{|c|}{$1988^{*}$} \\
\hline & Área & $\mathrm{kg} / \mathrm{ha}$ & Área & kg/ha \\
\hline El Salvador & 245 & 1614 & 278 & 2140 \\
\hline Guatemala & 563 & 1180 & 651 & 2110 \\
\hline Honduras & 350 & 1030 & 363 & 1500 \\
\hline Nicaragua & 261 & 800 & 220 & 1600 \\
\hline Costa Rica & 41 & 1000 & 61 & 1700 \\
\hline Panamá & 74 & 730 & 78 & 1500 \\
\hline Haití & 150 & 600 & 157 & 900 \\
\hline Rep. Dominicana & 35 & 1100 & 40 & 1600 \\
\hline Cuba & 80 & 1200 & 77 & 1300 \\
\hline Total & 1799 & 1028 & 1925 & 1594 \\
\hline
\end{tabular}

* Area expresada en miles.

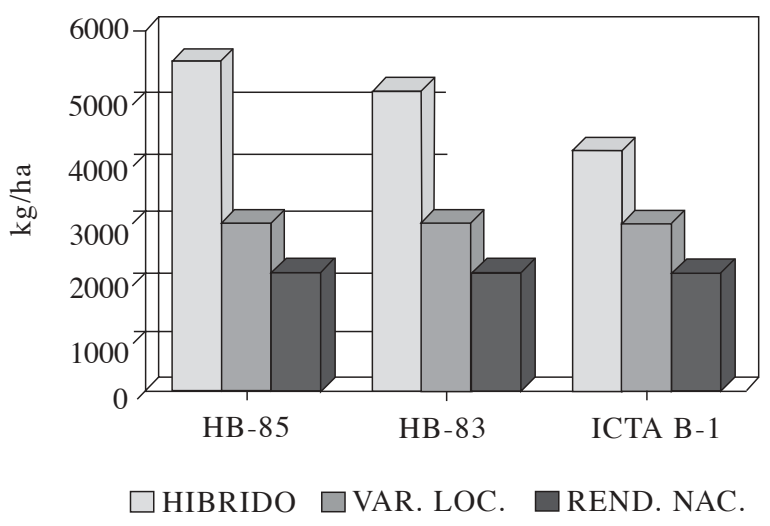

Figura 3. Progreso en el fitomejoramiento de maíz en Guatemala 1990.

\section{INVESTIGACIÓN EN TECNOLOGIA Y PRO- DUCCIÓN DE SEMILLA}

El progreso alcanzado en los últimos cinco años en el desarrollo de cultivares de maíz con alto potencial de rendimiento y tolerancia a factores adversos bióticos y abióticos ha motivado a que los programas nacionales de Centroamérica fortalezcan sus unidades o disciplinas de producción de semilla básica, para garantizar la producción de semilla certificada y que los agricultores se beneficien de las bondades de los nuevos cultivares. El éxito de un programa de semillas depende de un sistema confiable de aprovisionamiento continuo de semilla fundación de alta calidad para híbridos y de variedades de polinización libre, que garanticen la continuidad y larga vida de los cultivares liberados por el fitomejorador.

La producción de las categorías de semilla genética y fundación son responsabilidad del sector público y las categorías de semilla certificada corresponden a la empresa privada. Por esta razón se han establecido las unidades de semilla fundación que tienen el objetivo de producir y mantener la calidad de la semilla y desarrollar y demostrar tecnologías que faciliten la producción de semilla certificada por la industria regional de semillas.

La productividad de los cultivos modernos depende en gran medida de la calidad de semilla que el agricultor utiliza; una excelente calidad, es una característica diferencial que establece la brecha entre "semilla" y el material que los agricultores utilizan para siembra y en él basan la productividad y el sustento de sus familias.

Los agricultores cuyas áreas de producción se encuentran aisladas en laderas con suelos pobres, generalmente no tienen acceso a la semilla proveniente de los sistemas convencionales de producción de semilla, ya 


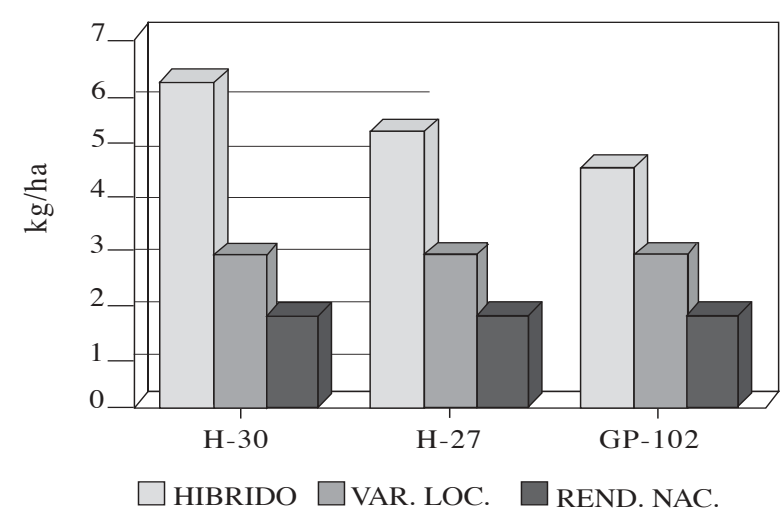

Figura 4. Progreso en el fitomejoramiento de maíz en Honduras 1990.

sea porque no son sujetos de crédito o porque la industria semillera no se interesa por esas áreas ya que las ganancias no son atractivas (Córdova 1990). Para suplir esta necesidad se han desarrollado los sistemas no convencionales de acondicionamiento de semillas, tales como la semilla artesanal.

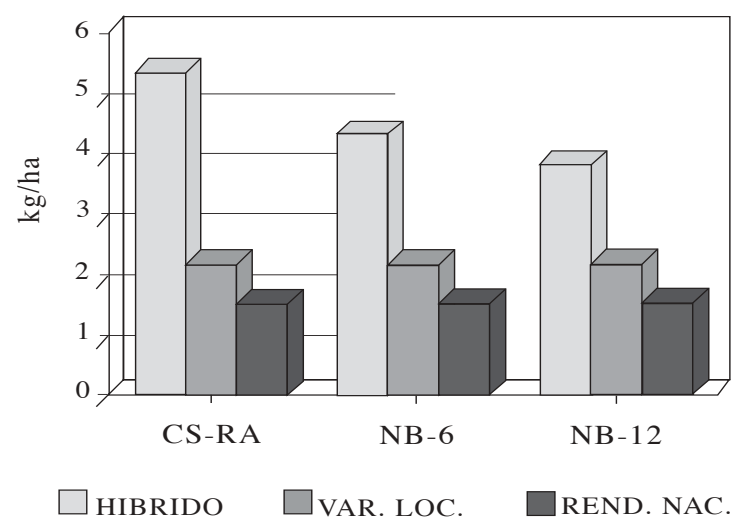

Figura 5. Progreso en el fitomejoramiento de maíz en Nicaragua 1990.

\section{Progreso en la investigación}

La investigación en "tecnología de producción de semillas" ha resuelto muchas incógnitas y ha producido tecnologías, las cuales fueron inmediatamente adoptadas por los productores de semilla en Guatemala, El Salvador y Honduras.

Es notable la ventaja comparativa que los productores obtienen al adoptar las nuevas prácticas. El cambio de la relación cuatro hembras y un macho por cua-

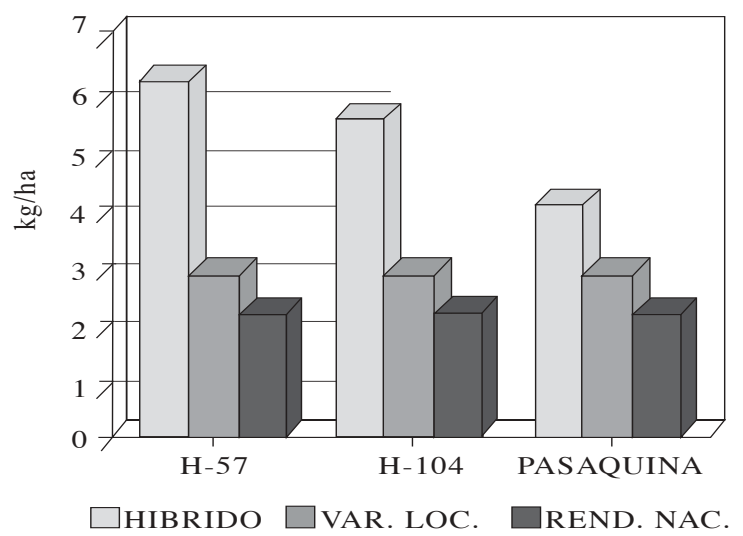

Figura 6. Progreso en el fitomejoramiento de maíz en El Salvador 1990.

tro hembras y un macho compacto, cosecha temprana (madurez fisiológica), descogolle con dos hojas en lugar de desespigue normal significa un incremento en rendimiento de semilla de aproximadamente del 30\% y disminución en $66 \%$ en los costos de desespigue.

\section{Semilla genética y básica}

La organización en las Unidades de Semilla Fundación ha permitido un notable avance en el abastecimiento oportuno y suficiente de semilla fundación de alta calidad. Por otra parte, se han logrado establecer sistemas ordenados y eficientes de mantenimiento y producción de semilla básica que permitirá y garantizará la vida y pureza genética de los cultivares liberados.

El Salvador, Guatemala, Honduras y Nicaragua han adoptado el sistema de mantenimiento escalonado de producción y mantenimiento de semilla de fundación de progenitores y variedad de polinización libre.

En 1990 se produjo el $42 \%$ de semilla de fundación más que 1989. El nuevo método de mantenimiento y producción de semilla genética a través de una sola generación eliminará las probabilidades de contaminación que ocurren en los sistemas de producción continua (Cuadro 6).

\section{Semilla Certificada}

La industria semillista de Centroamérica atraviesa por una crisis, congruente con la recesión global existente en todo el mundo. Sin embargo, se espera que en 1991 las medidas de estímulos de la producción de granos básicos ayuden a garantizar la seguridad alimentaria ya que permitiría a los productores de semilla nego- 
Cuadro 6. Producción de semilla certificada de maíz (Tm) en Centroamérica y El Caribe 1989 y 1990.

\begin{tabular}{lrr}
\hline \multicolumn{1}{c}{ País } & $\mathbf{1 9 8 9}$ & $\mathbf{1 9 9 0}$ \\
\hline El Salvador & 2272 & 2500 \\
Guatemala & 2000 & 2272 \\
Honduras & 1000 & $1136 *$ \\
Nicaragua & 1800 & 1500 \\
Costa Rica & 100 & 200 \\
Panamá & 363 & 400 \\
Rep, Dominicana & 100 & 100 \\
Haití & 50 & 50 \\
Cuba & 1200 & 1200 \\
TOTAL & 8885 & 9358 \\
\hline
\end{tabular}

* Incluye la producción de 681 y 450 toneladas métricas de los híbridos de DeKalb y Seminal respectivamente.

ciar sus excedentes y pasar a manos de los agricultores las 9358 toneladas de semilla de maíz de alta calidad que representan un 10\% más de la producción de 1989.

\section{CAMBIOS EN LOS SISTEMAS NACIONALES}

\section{Deterioro de los sistemas nacionales}

La falta de recursos económicos para financiar las actividades de generación y transferencia de tecnología propició el debilitamiento de los sistemas nacionales de investigación y extensión agrícola Este proceso se inició a mediados de la década de los 80 's y se acentuó al principio de los 90's, continuando hasta el presente la

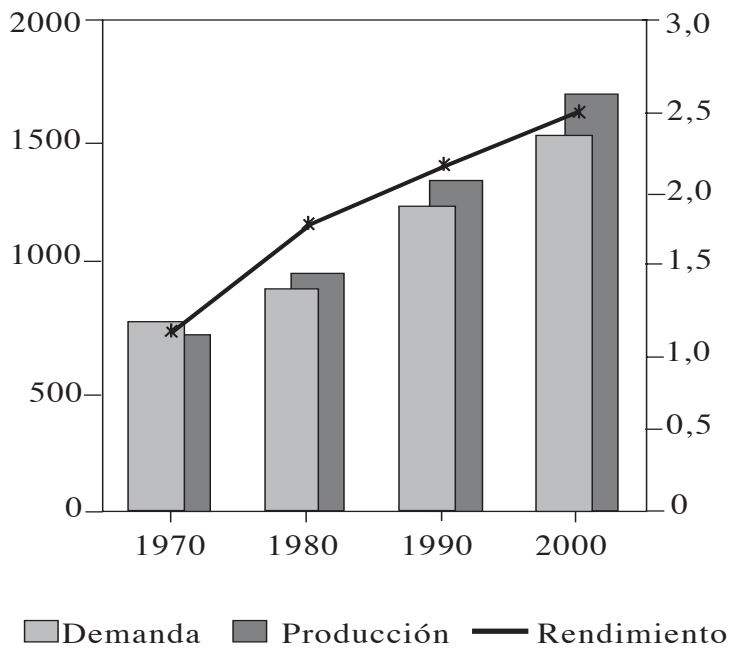

Figura 7. Estimadores de la producción en Guatemala. crisis de los organismos nacionales que ha afectado el desarrollo de actividades, a tal grado que el $75 \%$ de las instituciones de investigación en Centroamérica, sólo tienen presupuesto nacional para pagar salarios, por lo que muchos de los proyectos de investigación agrícola se conducen con el apoyo de instituciones internacionales ONG's.

La falta de estímulos a los investigadores de los programas nacionales propició la fuga de personal calificado al final de los 80 's cuando la empresa privada tenía oportunidad de empleo; en el presente, la crisis también ha alcanzado a las empresas privadas. Muchos de los investigadores en los programas nacionales tienen que complementar sus salarios con consultorías fuera de las instituciones de trabajo, lo que ha afectado el proceso de investigación y transferencia de tecnología y como consecuencia de este deterioro, la liberación de cultivares en el quinquenio (1991-1995) ha sido prácticamente nula.

\section{Cambio de estructura}

En la búsqueda de una solución a la crisis planteada anteriormente, algunos centros nacionales de investigación agrícola han acogido la propuesta del Banco Mundial, la cual promueve la reestructuración de la investigación y la extensión creando institutos autónomos de generación, prueba y transferencia de tecnología, en un esfuerzo integrado para resolver los problemas de la productividad en el campo. En la nueva estructura, las actividades de investigación están descentralizadas y la integración ocurre desde el nivel del campo.

Las perspectivas de esta nueva organización son prometedoras, ya que existen algunos buenos ejemplos: El nuevo CENTA y muy pronto INTA, sin embargo, muchos buenos investigadores cuya escolaridad no permitía formar parte de éstas nuevas instituciones quedaron en el camino.

Es evidente que el fortalecimiento de las nuevas instituciones tiene que contar con nuevos estímulos para los investigadores; al menos en el CENTA, ya se vislumbra un buen potencial para entrenamiento a nivel superior y los salarios han sido mejorados. Esperamos que el fortalecimiento sea real en todos los países antes que ocurra el cambio de siglo.

\section{Falta de apoyo en el campo}

Es evidente que la crisis de la globalización ha alcanzado a la región centroamericana, afectando en diferentes formas la productividad agrícola de la región al 
reducir la inversión, principalmente en la producción de granos básicos que son componente esencial en la garantía de la seguridad alimentaria.

Si los países centroamericanos se dicen eminentemente agrícolas y que poseen una ventaja comparativa en la producción de alimentos, es justo que esa ventaja sea explotada garantizando créditos oportunos y suficientes para eliminar las importaciones.

\section{ESTRATEGIAS HACIA EL AÑO 2000}

\section{Resistencia a factores adversos bióticos y abióticos}

No existe ninguna duda de que en la próxima década los factores adversos bióticos y abióticos limitantes de la producción, continuarán causando pérdidas substanciales y disminuirán los rendimientos de maíz, principalmente en las áreas marginales. Estos factores prioritarios que fueron identificados hace diez años, han sido atacados fuertemente y se ha logrado un progreso notable, principalmente en lo relativo a resistencia a achaparramiento (causado por virus y micoplasmas) y tolerancia a la sequía; sin embargo, el problema de pudrición de mazorca sigue siendo prioridad número uno. En este caso debe modificarse el proyecto orientándolo a la formación de híbridos y sintéticos a través del método de selección genealógica (pedigree breeding) y reciclaje de poblaciones $\mathrm{F}_{2}$ derivadas de cruzas élite resistente por resistente, haciendo autofecundaciones sucesivas hasta $\mathrm{S}_{4}$ por dos probadores élite, grupo heterótico A y grupo heterótico $\mathrm{B}$, sirviendo como probadores las líneas CML-247 y CML-254. La evaluación de estas cruzas se haría en ambientes adversos de pudrición tales como Cuyuta, Guatemala, Valle de Jamatran, Honduras y Los Diamantes en Costa Rica. Debe también lograrse un buen sistema de inoculación para pudrición de mazorca, al mismo tiempo que se evalúan las

Cuadro 7. Rendimiento de híbridos precoces en tres localidades de México, 1995.

\begin{tabular}{lrrrr}
\hline & t/ha & Dm & Khd & Mp\% \\
\hline P5xP8 & 9,21 & 114 & 81 & 4,1 \\
P2xP7 & 9,07 & 124 & 73 & 4,8 \\
P7xP8 & 8,99 & 120 & 75 & 5,1 \\
P2xP10 & 8,68 & 114 & 76 & 4,2 \\
A-7520 & $\underline{7,37}$ & $\underline{129}$ & $\underline{57}$ & 21,9 \\
MDS .05 & 1,63 & 6 & -- & 6,5 \\
\hline
\end{tabular}

Dm = Días a madurez fisiológica, Khd = kg/ha por día; $\mathrm{Mp}=$ Mazorcas podridas. cruzas de prueba se avanza la endogamia en las líneas y éstas se evalúan. En otras palabras, usar el "poder de la endogamia" para eliminar esos genes recesivos deleterios que limitan el progreso de la selección y a fijar la frecuencia de estos alelos favorables que determinan la resistencia a pudrición de mazorca; en este proceso se pueden formar progenitores híbridos y componentes de variedades sintéticas. Al logro de estos objetivos se tendrán híbridos y variedades sintéticas que reducirán las pérdidas post-cosecha debido a pudriciones.

\section{Híbridos eficientes y estables}

El proyecto de desarrollo de híbridos del PRM ha generado nuevos híbridos con buen potencial de rendimiento, sin embargo, no ha sido agresivo en los últimos cinco años para liberar nuevos híbridos que sirvan a todos los países de la región, manteniendo la particularidad en cada caso. Es obvio que los países más avanzados en hibridación (El Salvador, Guatemala y Honduras) sean más beneficiados, ya que pueden hacer combinaciones de sus progenitores locales, con las líneas liberadas por CIMMYT, esta debe de ser una actividad dinámica y progresiva. Los programas nacionales que no tienen programas fuertes de hibridación, podrán beneficiarse directamente con los híbridos desarrollados por CIMMYT, un ejemplo clásico de esto es Panamá.

La estabilidad del rendimiento se logra a través de la selección en contra de factores bióticos y abióticos, para desarrollar progenitores resistentes a enfermedades e insectos, tolerantes a sequía y más eficientes en la utilización de nitrógeno. Al reducir el efecto de dichos factores, los híbridos evaluados en ambientes contrastantes, mostrarán más estabilidad del rendimiento reduciendo la interacción genotipo x ambiente. Las líneas superiores $(10 \%)$ de los proyectos poblacionales de resistencia a factores adversos bióticos y abióticos, deben ser sistemáticamente evaluadas y avanzadas por endogamia dentro del proyecto de híbridos, de esta manera se ensamblan integrándose eficientemente.

El PRM debe ser más agresivo en la liberación de híbridos y establecer en cada país un sistema cíclico de liberación de cultivares, estableciendo el ciclo de vida de cada híbrido desarrollado en comunicación estrecha con la industria de semillas.

\section{Híbridos precoces}

Los híbridos de maíz precoces pueden ser muy importantes en áreas de cultivo intensivo, en rotaciones donde el período para producir un cultivo alternativo es 
muy corto, en cultivos en relevos o en cultivos múltiples haciendo uso más eficiente de la tierra.

El mejor conocimiento actual de componentes fisiológicos asociados al rendimiento de maíz nos muestra una nueva avenida para seleccionar progenitores de híbridos precoces. El período de llenado de grano se encuentra altamente correlacionado positivamente con el rendimiento por lo tanto, se deben seleccionar progenitores de híbridos cuyo período transcurrido entre fecha de siembra y días a antesis sea corto y el período transcurrido entre floración y madurez sea más largo. El subprograma de maíz tropical de CIMMYT ha desarrollado líneas precoces que producen híbridos de 100 días a la cosecha; otra alternativa es cruzar líneas de madurez intermedia con líneas precoces, logrando híbridos precoces con buen rendimiento. Preciado et al. (1996), mostraron que híbridos entre progenitores precoces subtropical $\mathrm{x}$ tropical dieron origen a híbridos que alcanzaron su madurez fisiológica a los 100 días, el período de llenado fue de 54 días y la floración femenina fue de 46 días. El híbrido superior rindió 6,7 t/ha, el experimento fue sembrado en Poza Rica, Veracruz.

\section{Perspectivas de la biotecnología}

Mucho se ha dicho en las dos décadas pasadas acerca del potencial de la biotecnología para el desarrollo de cultivares resistentes a factores adversos bióticos y abióticos, sin embargo, hasta 1996 esos resultados no se han visto aplicados en los campos de los agricultores de las áreas tropicales y subtropicales.

Recientes publicaciones indican que un nuevo híbrido MAX-486 de CIBA GEIGY, resistente al gusano barrenador, estará en manos de agricultores de la faja maicera de Estados Unidos de Norte América en mayo de 1996. Desde la perspectiva del CIMMYT, las alternativas que se vislumbran en apoyo de los fitomejoradores convencionales de maíz en la próxima década, son dos: selección asistida por marcadores moleculares y huellas de ADN.

La selección asistida por marcadores genéticos moleculares

El uso de la selección asistida por marcadores moleculares ofrece muchas ventajas importantes a los mejoradores en el desarrollo de líneas endogámicas (selección genealógica o selección por pedigree) y selección recurrente; estas ventajas están basadas en los siguientes principios: a) Identificación de progenitores élite con características superiores (resistencia a insectos y/o enfermedades) para iniciar los cruzamientos. Esta iden- tificación se realiza extrayendo el ADN de hojas de la planta y marcando las regiones del genomio que contienen la característica de interés, esto permite identificar solamente los individuos que contienen dichas regiones para realizar los cruzamientos, lo cual se realiza al azar en el mejoramiento convencional, b) Reducción de la cantidad de trabajo y costos de operación, ya que no es necesario sembrar cantidades grandes de progenies, c) Combinar la resistencia identificada en cromosomas diferentes y líneas diferentes, en una sola línea, d) Reducir el tiempo en el desarrollo de líneas o en el proceso de selección recurrente; esto se logra al obtener homocigosis en un sólo ciclo a través de rescate embrionario y desarrollo de la planta en cultivo de tejidos.

\section{Desarrollo de productos transgénicos}

El CIMMYT adquirió una fuente de resistencia a barrenadores en una línea homocigótica, conteniendo el gene Bt, esta fuente fue incorporada por cruza y retrocruza normal en líneas subtropicales y tropicales del CIMMYT.

Se espera que en el futuro se puedan obtener híbridos resistentes a barrenador y cogollero, el principio utilizado en la resistencia es el mismo usado para producir insecticidas que controlan insectos de lepidópteros principalmente.

\section{Políticas conservacionistas del ambiente}

Desarrollar germoplasma resistente a factores adversos bióticos y abióticos que reduzcan el riesgo de los agricultores en los ambientes marginales en inversiones en otros insumos agrícolas que necesitan también del apoyo de políticas protectoras del medio ambiente y tecnología de conservación del recurso base, por lo tanto, es urgente que en Centroamérica se establezcan leyes que protejan los suelos de la erosión, evitando el uso desmedido de quemas de rastrojo y aplicación de sistema de labranza de conservación, en pocas palabras establecer una ley en contra de la quema de rastrojos.

\section{Industria de semillas agresiva y dinámica}

La industria de semilla (empresas productoras privadas y sector público) debe establecer una posición dinámica y agresiva en todas las etapas del proceso, desde la liberación, demostración y producción de la semilla.

Las siguientes consideraciones son necesarias en la próxima década, partiendo de las premisas siguientes: 
a) Existen nuevos agricultores, existen nuevas oportunidades, como consecuencia de esto deben realizarse promociones masivas que demuestren al agricultor las ventajas de los nuevos cultivares, b) La industria semillista con sus distribuidores debe participar activamente en la promoción de nuevos híbridos usando todos los medios modernos de promoción, c) Debe establecerse el ciclo de vida de los híbridos liberados disminuyendo el sentido paternalista al agricultor, para que no pasen 30 años para ofrecerle un nuevo cultivar, lo cual significa pérdidas anuales para el agricultor del orden del $20 \%$.

\section{Apoyo a los sistemas nacionales}

Los sistemas nacionales de investigación deben de recibir el apoyo del sector público y del sector privado de acuerdo a la consideración de la tasa retorno a capital por el producto desarrollado por la investigación, los ejemplos de Brasil y Egipto son muy claros en el pago de regalías y venta de semilla básica (valor adicional al costo) como retribución al desarrollo de los cultivares desarrollados.

\section{LITERATURA CITADA}

AGUILUZ, A.; URBINA, R.; CELADO, R.; CÓRDOVA, H. 1990. Efecto y mejoramiento para resistencia al acapa- ramiento sobre el rendimiento de cultivares de maíz evaluados en siete ambientes de Centroamérica y El Caribe. In: Síntesis de resultados experimentales. Programa Regional de Maíz para Centro América, Panamá y El Caribe. 19p.

BRIZUELA, L.; MARADIAGA, N.; ZEA, J.; AGUILUZ, A.; CÓRDOVA, H. 1990. Respuesta a selección recurrente a familias de medios hermanos de maíz (Zea mays L.) adaptadas al ambiente de humedad limitada. Programa Regional de Maíz para Centro América, Panamá y El Caribe. 22p.

CÓRDOVA, H. 1990. Desarrollo y mejoramiento de germoplasma para resistencia a factores adversos bióticos y abióticos y producción de semillas. Estrategias y logros 1986-1991. In: Síntesis de resultados experimentales. Programa Regional de Maíz para Centroamérica, Panamá y El Caribe.

CÓRDOVA, H.S.; BARRETO, H.J.; CROSSA, J. 1996. Maize Hybrid Development in Central America: Reliability of Yield Gains Against a Regional Check. Maydica (41) 349:353.

JIMÉNEZ, K.; SALAS, C.; CALDERÓN, C.; PIXLEY, L. 1990. Avances del Proyecto Colaborativo Regional de Mejoramiento Integral para Cobertura. Pudrición de mazorca y rendimiento de maíz. Costa Rica. In: Síntesis de resultados experimentales. Programa Regional de Maíz para Centroamérica, Panamá y El Caribe. 18p. 